Notre Dame Journal of Formal Logic

Volume VIII, Number 4, October 1967

\title{
ON THE EXTENSION OF S4 WITH CLMPMLP
}

\author{
R. A. BULL
}

Over the last few years various logicians have considered the modal system obtained by extending $\mathrm{S}^{1}$ with

\section{$C L M p M L p$,}

but no demonstration that the system is decidable, or description of a characteristic model for it, has been published. ${ }^{2}$ The purpose of this paper is to fill this gap by showing the system to have the finite model propertyso that it is decidable (by [2], Lemma 4) and characterized by order closure models (by [1], Lemma 1)-and obtaining a characteristic order closure model for it. I assume familiarity with closure algebras (see [3] and [4]), with the order closure models of [1], and with the finite model property (see [2]). I do not distinguish between a closure algebra and the model obtained from it by designating the unit element; a closure algebra can be regarded as a Boolean algebra with a closure operator defined on it, and this representation is the most convenient for my purposes. I use the symbol - for relative complement, instead of in its normal role of complement proper; and I use the interior operator I (complement of closure of complement).

(It may be of interest that the system can also be obtained by extending S4 with either of the rules

$$
\begin{aligned}
& \vdash M \alpha \Longrightarrow \vdash M L \alpha \\
& \vdash M \alpha, \vdash M \beta \Longrightarrow \vdash M K \alpha \beta .
\end{aligned}
$$

To prove this I derive them in rotation:

$$
\begin{aligned}
\text { (a) Given } & C L M p M L p, \\
\vdash M \alpha & \Longrightarrow \vdash L M \alpha \\
& \Longrightarrow \vdash L M \alpha, \vdash C L M \alpha M L \alpha \\
& \Rightarrow \vdash M L \alpha .
\end{aligned}
$$

(b) Given $\vdash M \alpha \Longrightarrow \vdash M L \alpha$, since $\vdash_{s_{4}} C L M L p C L M L q M K p q$, 


$$
\begin{aligned}
\vdash M \alpha, \vdash M \beta & \Longrightarrow \vdash M L \alpha, \vdash M L \beta \\
& \Longrightarrow \vdash L M L \alpha, \vdash L M L \beta \\
& \Longrightarrow \vdash L M L \alpha, \vdash L M L \beta, \vdash C L M L \alpha C L M L \beta M K \alpha \beta \\
& \Longrightarrow \vdash M K \alpha \beta .
\end{aligned}
$$

(c) Given $\vdash M \alpha, \vdash M \beta \Longrightarrow \vdash M K \alpha \beta$, since $\vdash_{s 4} C M C p q C L p M q$, $\vdash_{s_{4}} M C M p p, \vdash_{s 4} M C M p C p L p \Longrightarrow \vdash M K C M p p C M p C p L p$

$$
\begin{aligned}
& \Longrightarrow \vdash M C M p K p C p L p \\
& \Longrightarrow \vdash M C M p L p \\
& \Longrightarrow \vdash M C M p L p, \vdash C M C M p L p C L M p M L p \\
& \Longrightarrow \vdash C L M p M L p .)
\end{aligned}
$$

In what follows I shall refer to the Lindenbaum algebra of equivalence classes of words in the system as $\mathfrak{m ( = \langle B , C}\rangle)$. This is known to be a closure algebra characterising the system-see Theorem 3.6 of [4]. In the next three paragraphs I show how to embed a given finite fragment of $\mathfrak{i}$ in a finite closure algebra which verifies the system. For each non-thesis of the system, construct such a closure algebra on the values of the parts of the non-thesis in a rejection with $\mathbb{m}$ : clearly the system has the finite model property with these models.

Following [3], for any finite sub-set $Y$ of the elements of $\mathfrak{M}$, I define $\mathfrak{M}_{y}=\left\langle\mathbb{B}_{y}, \mathrm{C}_{y}\right\rangle$ as follows:

(1) $B_{y}$ is the (finite) sub-Boolean algebra of $B$ generated by $Y$.

(2) $C_{y}$ is the function on $B_{y}$ given by $C_{y} x=\bigcup_{i \in I} y_{i}$, where $\left\{y_{i} \mid i \varepsilon I\right\}$ is the set of all elements $y$ of $\mathfrak{B}_{y}$ such that $x \subseteq y=C y$.

Given a finite sub-set $X$ of the elements of $\Re$, I use $a_{x}$ for the element of given by

$$
a_{x}=\bigcup_{x \in M_{x}}(C x-x),
$$

take $X^{\prime}=X \cup\left\{a_{x}\right\}$, and define an algebra $\mathbb{m}_{x}^{1}=\left\langle B_{x}^{1}, C_{x}^{1}\right\rangle$ by

(3) $B_{x}^{1}=B_{x}$

(4) $C_{y}^{1}$ is the function on $B_{x}^{1}$ given by $C_{x}^{1} x=\left(x-a_{x}\right) \cup\left(C_{x^{\prime}} x \cap a_{x}\right)$.

$m_{y}$ is known to be a closure algebra-see Lemma 2.3 of [3]-and $m_{x}^{1}$ can be shown to be a closure algebra by straightforward applications of the properties of,$M_{x^{\prime}}$. (In showing that $C_{x}^{1} C_{x}^{1} x=C_{x}^{1} x$, note that $C_{x^{\prime}} \mathbf{C}_{x}^{1} x=C_{x^{\prime}} x$, since $\mathrm{C}_{x^{\prime}} x$ contains $\mathrm{C}_{x^{\prime}}\left(x-a_{x}\right)$ and $\mathrm{C}_{x^{\prime}}\left(\mathrm{C}_{x^{\prime}} x \cap a_{x}\right)$.)

The properties of $\mathfrak{M}_{x^{\prime}}$ can also be used to check that

$$
\mathrm{I}_{x}^{1} x=\left(x-a_{x}\right) \cup\left(\mathrm{I}_{x^{\prime}} x \cap a_{x}\right)
$$

Using this we find that $I_{x}^{1} a_{x}$ is $\wedge$; for

$$
\begin{aligned}
& \mathrm{I}(\mathrm{C} x-x)=\wedge \text { in all closure algebras; } \\
& \therefore \mathrm{I} \bigcup_{x \in \mathbb{M}_{x}}(\mathrm{C} x-x)=\wedge \text { in } \mathbb{M} \text {, using the strong verification of the rule } \vdash M \alpha \text {, } \\
& \vdash M \beta \Longrightarrow \vdash M K \alpha \beta \text { in } M \text {; } \\
& \therefore l a_{x}=\Lambda \text {, by the definition of } a_{x} \text {; } \\
& \therefore I_{x^{\prime}} a_{x}=\wedge \text {, using the properties of } \mathrm{m}_{x^{\prime}} \text {; } \\
& \therefore \mathrm{I}_{x}^{1} a=\left(a_{x}-a_{x}\right) \cup\left(\mathrm{I}_{x} a_{x} \cap a_{x}\right)=\wedge \text {. }
\end{aligned}
$$


I can now show that $\mathfrak{M}_{x}^{1}$ verifies $\vdash M \alpha \Longrightarrow \vdash M L \alpha$, and so the system; for if $\mathrm{I}_{x}^{1} x$ is $\wedge$ then $\left(x-a_{x}\right)$ is $\wedge$, and

$$
\begin{aligned}
\mathbf{I}_{x}^{1} \mathbf{C}_{x}^{1} x & =\mathbf{I}_{x}^{1}\left(\left(x-a_{x}\right) \cup\left(\mathbf{C}_{x^{\prime}} x \cap a_{x}\right)\right) \\
& =\mathbf{I}_{x}^{1}\left(\mathbf{C}_{x^{\prime}} x \cap a_{x}\right) \\
& \subseteq \mathbb{I}_{x}^{1} a_{x},
\end{aligned}
$$

so that $I_{x}^{1} C_{x}^{1} x$ is $\wedge$.

I must finally show that the fragment of $M$ with elements $X$ is embedded in $\mathfrak{M}_{x}^{1}$. That it is embedded qua Boolean algebra follows immediately from definitions (1) and (3). It remains to show that if $x$ and $C x$ are in $X$ then $C_{x}^{1} x$ is $C x$. It is known that in this case $C_{x^{\prime}} x$ is $C x$ (see Lemma 2.3 of [3]); therefore

$$
\begin{aligned}
C_{x}^{1} x= & \left(x-a_{x}\right) \cup\left(C_{x^{\prime}} x \cap a_{x}\right) \\
= & \left(x-a_{x}\right) \cup\left(\mathbf{C} x \cap a_{x}\right) \\
= & \left(x-a_{x}\right) \cup\left((x \cup(\mathbf{C} x-x)) \cap a_{x}\right) \\
= & \left(x-a_{x}\right) \cup\left(x \cap a_{x}\right) \cup\left((\mathbf{C} x-x) \cap a_{x}\right) \\
= & x \cup(\mathbf{C} x-x), \text { since } a_{x} \supseteq \mathbf{C} x-x \text { by the definition of } a_{x} \text { and the hy- } \\
& \text { pothesis that } x \text { is in } X, \\
= & \mathbf{C} x .
\end{aligned}
$$

We now know that the system is characterized by order closure models, and the rest of the paper is devoted to discussing them. For this I must add some notation to that of [1]. (Also, on one point I wish to alter that of [1]: the construction on quasi-ordered sets, given on p. 253 of [1], which is represented by a subscript 1 , shall be represented by a superscript 1 here.) I use the term bottom point for a member $a$ of a quasiordered set for which $a \geqslant x$ only if $a \leqslant x$, and use the term strict bottom point for a member $a$ of a quasi-ordered set for which $a \geqslant x$ only if $a=x$. I use $\Omega^{-}$for the quasi-ordered set obtained from $\Omega$ by deleting its strict bottom points, and use $\AA^{b}$ for the quasi-ordered set obtained from $\Omega$ by adding a point strictly below each bottom point.

It is easily checked that a finite order closure model verifies the system if and only if each bottom point of the quasi-ordered set on which it is defined is a strict bottom point. Here, then, is a set of models characterizing the system. Following Example 1 of [1], I shall use this to show that $\mathcal{S}^{1 b+}$ (for $\mathcal{S}^{1}$ see the third paragraph of p. 258 of [1]) is a characteristic model for the system. It is easily checked that this model verifies the system, so it remains to show that every word rejected by a finite model of the kind described is rejected by $\delta_{2}^{1 b+}$. First I need the following variant of Lemma 5 of [1].

Lemma. If $\Re$ is a finite quasi-ordered set such that $\AA^{+}$is a model for the system, and if $\left\{\right.$ is a partially ordered set such that $\mathfrak{R}^{-e+}$ is isomorphic to a subalgebra of $\Omega^{+}$, then $\Omega^{+}$is isomorphic to a subalgebra of $\Omega^{1 b+}$

Proof. Let $\Phi$ be the isomorphism between $\Omega^{-e+}$ and a subalgebra of $\Omega^{+}$. I define a mapping $\Psi$ of $\AA$ onto $\Omega^{1 b}$ in two steps: 
(1) If $\left\{a_{0}, a_{1}, \ldots, a_{n}\right\}$ is an equivalence class of members of $\AA^{-}$then

$\Psi a_{i}=\left\{\langle b, i>| b \varepsilon \Phi\left\{a_{0}\right\}\right\}$, for $0 \leqslant i \leqslant n-1$, $\Psi a_{n}=\left\{\langle b, j\rangle \mid b \varepsilon \Phi\left\{a_{0}\right\}, j \geqslant n\right\}$.

(2) If $a_{0}, a_{1}, \ldots, a_{n}$ are strict bottom points immediately below a point $b$, and $D$ is the set of those bottom points of $\{$ which are in $\Phi\{b\}$, then

$$
\begin{aligned}
& \Psi a_{i}=\{\langle c, i\rangle|\langle c, i\rangle<<d, i\rangle \text { for } d \varepsilon D\}, \text { for } 0 \leqslant i \leqslant n-1, \\
& \Psi a_{n}=\{\langle c, j\rangle|\langle c, j\rangle<<d, j\rangle \text { for } d \varepsilon D, j \geqslant n\} .
\end{aligned}
$$

(In both cases, when $n$ is 0 apply the second clause to $a_{0}$.) Mapping sets of points of $\Omega$ onto the unions of their images under $\Psi$ gives the required isomorphism.

Now suppose we are given a non-thesis of the system. We know that this is rejected by a finite order closure model, $\Omega^{+}$say, for the system. We may take $\Omega^{-e}$ to have a greatest point, since a word rejected by an order closure model must be rejected by the order closure model on the sub-tree of points below a point in a non-empty value of the word in the original model. By Lemmas 3 and 10 of [1], $\Omega^{-e+}$ is isomorphic to a subalgebra of $\mathscr{S}_{i}^{+}$(for $\mathscr{S}_{i}$ see the first paragraph of p. 258 of [1]) for some $i$. Therefore, by the Lemma given above, $\mathfrak{R}^{+}$must be isomorphic to a subalgebra of $\mathcal{S}_{i}^{1 b+}$; so the given non-thesis is rejected by $\mathcal{S}_{2}^{1 b+}$. So the given non-thesis is rejected by $\mathcal{S}^{1 b+}$, using any allocation which copies a rejecting allocation in $\mathcal{S}_{i}^{1 b+}$ in a sub-tree of depth $i+1$.

\section{NOTES}

1. In this paper I take $S 4$, and extensions of it, to be given with $\vdash \alpha \Longrightarrow \vdash L \alpha$ as a derivation rule.

2. While in Oxford in 1963, Kripke mentioned some very interesting results for this system with predicates and quantifiers, so I would not be surprised if he has also obtained the results given here.

Added 18-8-65: It is pointed out to me that this system was first put forward by McKinsey, in 1945, and that systems containing CLMPMLP are being examined by Prior, Sobocinski, and Ivo Thomas in current numbers of the Notre Dame Journal of Formal Logic. See the discussion in [5].

\section{REFERENCES}

[1] Dummett, M. A. E., and E. J. Lemmon, Modal logics between S4 and S5. Zeitschrift fur Mathematische Logik und Grundlagen der Mathematik, Bd 5 (1959), pp. 250-264.

[2] Harrop, R., On the existence of finite models and decision procedures for propositional calculi. Proc. Camb. Phil. Soc., vol. 54 (1958), pp. 1-13.

[3] McKinsey, J. C. C., and Alfred Tarski, The algebra of topology, Annals of Math., vol. 45 (1944), pp. 141-191. 
[4] McKinsey, J. C. C., and Alfred Tarski, Some theorems about the sentential calculi of Lewis and Heyting. The Journal of Symbolic Logic, vol. 13 (1946), pp. 1-15.

[5] Sobocinski, B., Remarks about the axiomatization of certain modal systems. Notre Dame Journal of Formal Logic, v. V (1964), pp. 71-80.

\section{Wadham College}

Oxford, England 